\title{
A randomized controlled trial of ketorolac for prevention of headache related to electroconvulsive therapy
}

\author{
Keith G. Rasmussen \\ Mayo Clinic Department of Psychiatry and Psychology, Rochester, USA; rasmussen.keith@mayo.edu
}

Received 15 April 2013; revised 19 May 2013; accepted 10 June 2013

Copyright (c) 2013 Keith G. Rasmussen. This is an open access article distributed under the Creative Commons Attribution License, which permits unrestricted use, distribution, and reproduction in any medium, provided the original work is properly cited.

\begin{abstract}
Background and Purpose: Headache is one of the most common side effects of electroconvulsive therapy (ECT), with a reported prevalence as high as $45 \%$. Typical pharmacologic measures include aspirin, acetaminophen, or nonsteroidal anti-inflammatory medications. Among the latter, ketorolac may be especially advantageous in that it can be administered intravenously right before a treatment. The primary aim of this study was to measure the efficacy of intravenous ketorolac administration for the prevention of post-ECT headache at the first treatment session. Methods: Sixteen patients were assigned to the control group, while eight patients were assigned to the ketorolac treatment group (8 males, 16 females; mean age \pm standard deviation $=46 \pm 13.5$ years). Statistical analysis consisted of a one-way analysis of variance using the two-sample test. We utilized a post-ECT headache severity scale from zero (no headache) to 3 (severe headache). Results: The mean score for the control group was $1.3( \pm 1.1)$, while the mean score for the ketorolac treatment group was 1.2 ( \pm 1.1$), p=0.86$ (not significant). Conclusions: Ketorolac administration does not decrease the incidence of post ECT headache at the first treatment session. It is possible that ketorolac may be effective at subsequent treatments for patients with particularly bothersome headaches after the first treatment. Implications: Ketorolac should not be routinely used at the first treatment session to prevent headache associated with ECT.
\end{abstract}

Keywords: Electroconvulsive Therapy; Ketorolac; Headache

\section{INTRODUCTION}

ECT is a commonly administered treatment for serious psychiatric illnesses such as depression, mania, and nonaffective psychosis [1]. It is often used to treat patients who are unresponsive to medication, those who are unable to tolerate the side effects associated with medication or those patients who need a more rapid response than medication alone can provide [1].

Treatment is performed under general anesthesia and involves the application of a brief electrical current to the brain. The electrical current excites neuronal cells and results in the production of a tonic clonic seizure which usually lasts 20 to 60 seconds. Treatments typically take place three times a week for a total of two to four weeks [1].

Immediate side effects from ECT include headache, myalgias, nausea, and confusion, which usually occur during the first few hours following the procedure [1]. Headache and nausea are the two most common post treatment complaints, with the reported prevalence of post ECT headache as high as 45\% [1,2]. Although the etiology of post-ECT headache is unknown, there are several theories. ECT results in an up-regulation of $5-\mathrm{HT}_{2}$ receptors [1]. Up-regulation and subsequent sensitization of $5-\mathrm{HT}_{2}$ receptors may trigger a vascular type headache [1]. ECT results in direct stimulation of the masseter, temporalis, and pterygoid muscles which is not attenuated by administration of succinylcholine. The associated jaw clamping may produce headache [1]. Other theories suggest that increased cerebral blood flow and blood pressure during ECT may contribute to the onset of headache [1].

The American Psychiatric Association (APA) suggests symptomatic treatment of post ECT headaches with aspirin, acetaminophen or non-steroidal anti-inflammatory medications including intravenous ketorolac [1]. The APA further suggests that patients who experience frequent 
post-ECT headache may benefit from symptomatic or prophylactic treatment with these medications [1]. Ketorolac is a non-steroidal anti-inflammatory drug providing relief from short-term acute pain. While the efficacy of ketorolac for post-operative pain management has been studied extensively, we have found no studies that assess its clinical effectiveness as a prophylactic treatment for the prevention of post-ECT headache.

Herein, we studied the efficacy of prophylactic intravenous ketorolac administration in randomized, blinded manner for the prevention of post ECT headaches utilizing a control group given no ketorolac. The primary aim of this study was to measure the efficacy of intravenous ketorolac administration for the prevention of post-ECT headache at the first treatment session.

\section{MATERIALS AND METHODS}

The study was approved by the Mayo Clinic Institutional Review Board. Patients were recruited from April, 2009, through March, 2010. Prior to their first ECT treatment at Mayo Clinic, informed consent was obtained from 29 patients at Mayo Clinic, Rochester. Thirteen of the study participants received ketorolac, while nineteen study participants did not. Patients were assigned to one of two treatments (ketorolac vs not) using a randomization schedule. The randomization schedule was prepared by the Division of Biomedical Statistics and Informatics and was stratified in blocks of four to ensure that after every fourth patient had been randomized, there were an equal number assigned to each treatment group. Individual randomization assignments were kept in sealed envelopes and numbered from one to twenty-nine, and only opened for a given patient after consent has been obtained. This ensured that everyone was blinded to the randomization schedule. Post ECT headache severity was then rated by each participant on a 4-point scale $(0=$ no headache, 1 = mild headache, 2 = moderate headache, 3 = severe headache).

The patient's pre-treatment care included placement of an intravenous catheter and administration of $0.2 \mathrm{mg}$ of intravenous glycopyrrolate to prevent vagal arrhythmias and excess oral salivation, unless it was contra-indicated. Patients received sodium thiopental for anesthesia induction. Study participants then randomly received the recommended dose of $30 \mathrm{mg}$ or $15 \mathrm{mg}$ of intravenous ketorolac after induction of general anesthesia. Following induction of general anesthesia, succinylcholine was administered for paralysis. Following muscle relaxation, the electrical stimulus was administered and the seizure ensued. Preoxygenation was given prior to induction of anesthesia. Once patients were apneic they were hand ventilated with Ambu-bag mask until spontaneous respirations resumed after the seizure. Patients were moni- tored with blood pressure, pulse oximetry, and continuous electrocardiogram (ECG). Following ECT administration, patients were transferred to the recovery area and were fully recovered, and sent to their inpatient rooms. Post ECT headache severity was then rated by each participant on a 4-point scale.

Patients 18 years of age or older providing their own consent for ECT were included. Ketorolac dosage adjustment from the usual $30 \mathrm{mg}$ to $15 \mathrm{mg}$ was made for patients at the age of 65 years or older or under $50 \mathrm{~kg}$. Patients not providing their own consent for ECT treatment were excluded. Patients who received outpatient ECT therapy were excluded. Patients with asthma, renal impairment (serum creatinine $>1.5 \mathrm{mg} / \mathrm{dL}$ ) or risk for renal failure due to volume depletion, suspected or confirmed cerebrovascular bleeding, hemorrhagic diathesis, incomplete hemostasis, high risk of bleeding, history of liver dysfunction, recent coronary artery bypass graft surgery (within the last month), and those allergic to nonsteroidal anti-inflammatory drugs were also excluded. Patients who presented to the ECT suite already complaining of a headache and those allergic to ketorolac were also excluded.

The patients and all outcome assessors were blinded to the administration of ketorolac. The trial operators, anesthesia providers and psychiatric staff in the ECT suite were not blinded. The outcome assessors included student registered nurse anesthetists, who enrolled and consented patients in the pre-treatment suite, collected demographic data from the treatment on the case report form, and gave the anesthesia provider the envelope containing that patient's randomization assignment.

The standardized questionnaire for post ECT headache severity was utilized by the study staff in the afternoon following ECT treatment. Additional demographic data collected included age, body mass index, gender, Anesthesiological Society of America status, and additional medications/anesthetics administered.

One-way analysis of variance using the two-sample test was the statistical analysis used to determine the headache rating.

\section{RESULTS}

Twenty-nine patients (10 males, 19 females) were enrolled in the study. Fifteen patients were assigned to the control group, while twelve patients were assigned to the treatment group. One patient withdrew from the study before ECT treatment, while another patient never received ECT treatment. Additionally, due to documentation discrepancies, it was undetermined if three patients randomized to ketorolac actually received that drug, so these patients were excluded from analysis. Thus, of 29 patients who signed the consent form, analyzable data 
are available for 24 (16 in the control group and 8 in the ketorolac group). All patients were medically healthy and free from serious illness such as cardiac or neurological disease.

Demographic and outcome data are presented in the Table 1. The mean headache severity score for the control group was $1.3( \pm 1.1)$, while the mean headache severity score for the treatment group was $1.2( \pm 1.1)$ with a $p$ value of 0.86 . In the one-way analysis of variance of age by drug group using the 2-sample test, the mean ages for the drug group were 45 years and the control group was 48 years $(p=0.6$, not significant). For a similar analysis of body mass index by group, the average body mass index for the drug group was 29 and for the control group was 31 ( $p=0.9$, not significant). The results of the one-way analysis of American Society of Anesthesiologists classification by group revealed a mean score for the drug group of 2.9 and for the control group of 2.9 (p $=0.8$, not significant). In a contingency analysis of gender by group using the Fisher exact 2 tail test, the p value was 0.4 (not significant). Of the eight patients who received ketorolac, four were male and four female. Of the 16 patients who were in the control group, four were male and twelve female.

\section{DISCUSSION}

Prophylactic ketorolac administration does not appear to prevent or decrease the severity of post ECT headache at the first treatment session. With a $\mathrm{p}$ value of 0.86 comparing the mean headache scores between the two groups, there was simply no signal whatsoever of a possible prophylactic benefit of ketorolac.

We should note that the study results as indicated pertain to the first treatment of a patient's series. We do not feel ketorolac should be used on a routine basis at the first treatment session. Further controlled trial research is indicated at subsequent treatments for those patients who experience headache after the first treatment.

Table 1. Demographic and outcome data.

\begin{tabular}{cccc}
\hline $\begin{array}{c}\text { Demographic/ } \\
\text { Outcome Variable }\end{array}$ & $\begin{array}{c}\text { Ketorolac } \\
\text { Group }\end{array}$ & $\begin{array}{c}\text { Control } \\
\text { Group }\end{array}$ & p value \\
\hline Gender Distribution & 4 males/4 females & 4 males/12 females & 0.4 \\
Age (mean) & 45 & 48 & 0.6 \\
ASA Score & 2.9 & 2.9 & 0.8 \\
$\begin{array}{c}\text { Body Mass Index } \\
\text { Headache Score } \\
\text { (mean } \pm \text { SD) }\end{array}$ & $1.2( \pm 1.1)$ & 31 & 0.9 \\
\hline
\end{tabular}

ASA = Anesthesiological Society of America; SD = standard deviation.
Traditional theories have attributed post ECT headache to succinylcholine associated myalgias as well as masseter pain associated with the delivery of the electrical current and subsequent clenching of the jaw. Based on these theories, treatment with ketorolac has been endorsed, but perhaps there is another mechanism at play.

Markowitz et al. [2], DeBattista and Mueller [3], and Oms et al. [4] published reports of severe post ECT headache which responded to sumatriptan. Sumatriptan is a serotonin (subtypes $1 \mathrm{~B}$ and $1 \mathrm{D}$ ) receptor agonist and is thought to relieve headaches by constricting cerebral arteries. Weiner et al. [5] suggested a serotonergic mechanism both for the efficacy of ECT and its tendency to produce headache. ECT up-regulates serotonin type 2 receptors, sensitization of which has been implicated in the onset of headaches. Studies testing the efficacy of prophylactic serotonin type 2 receptor antagonists for prevention of post ECT headache may prove helpful.

Perhaps changes in cerebral vasculature mediated by ECT contribute to post ECT headaches. Study of this proposed mechanism as well as a study of the efficacy of triptans for relief of post ECT headache is warranted.

\section{CONCLUSION}

Routine use of intravenous ketorolac is not indicated at the first ECT treatment session unless a patient has been treated with ECT in the past and experienced headaches in the post-treatment period.

\section{REFERENCES}

[1] American Psychiatric Association (2001) The practice of ECT: Recommendations for treatment, training, and privileging. American Psychiatric Press Inc., Washington DC.

[2] Markowitz, J.S., Kellner, C.H., DeVane, C.L., Beale, M.D., Folk, J., Burns, C. and Liston, H.L. (2001) Intranasal sumatriptan in post-ECT headache: Results of an open-label trial. The Journal of ECT, 17, 280-283. doi:10.1097/00124509-200112000-00008

[3] DeBattista, C. and Mueller, K. (1995) Sumatriptan prophylaxis for postelectroconvulsive therapy headaches. Headache, 35, 502-503. doi:10.1111/j.1526-4610.1995.hed3508502.x

[4] Oms, A., Miro, E. and Rojo, J. (1998) Sumatriptan was effective in electroconvulsive therapy (ECT) headache. Anesthesiology, 89, 1291-1292. doi:10.1097/00000542-199811000-00054

[5] Weiner, S.J., Ward, T.N. and Ravaris, C.L. (1993) Headache and electroconvulsive therapy. Headache, 34, 155159. doi:10.1111/j.1526-4610.1994.hed3403155.x 


\section{ABBREVIATIONS}

ECT = electroconvulsive therapy.

APA = American Psychiatric Association .

$\mathrm{Mg}=\operatorname{milligram}(\mathrm{s}) . \mathrm{Kg}=\operatorname{kilogram}(\mathrm{s})$. 